\title{
Resonance bifurcations from robust homoclinic cycles
}

\author{
Claire M Postlethwaite $\nmid \S$ and Jonathan H P Dawesł \\ $\dagger$ Department of Mathematics, University of Auckland, Private Bag 92019, Auckland \\ 1142, New Zealand \\ $\ddagger$ Department of Mathematical Sciences, University of Bath, Claverton Down, Bath \\ BA2 7AY, UK
}

\begin{abstract}
We present two calculations for a class of robust homoclinic cycles with symmetry $\mathbb{Z}_{n} \ltimes \mathbb{Z}_{2}^{n}$, for which the sufficient conditions for asymptotic stability given by Krupa and Melbourne are not optimal.

Firstly, we compute optimal conditions for asymptotic stability using transition matrix techniques which make explicit use of the geometry of the group action.

Secondly, through an explicit computation of the global parts of the Poincaré map near the cycle we show that, generically, the resonance bifurcations from the cycles are supercritical: a unique branch of asymptotically stable period orbits emerges from the resonance bifurcation and exists for coefficient values where the cycle has lost stability. This calculation is the first to explicitly compute the criticality of a resonance bifurcation, and answers a conjecture of Field and Swift in a particular limiting case. Moreover, we are able to obtain an asymptotically-correct analytic expression for the period of the bifurcating orbit, with no adjustable parameters, which has not proved possible previously. We show that the asymptotic analysis compares very favourably with numerical results.
\end{abstract}

AMS classification scheme numbers: 34C37, 37C80, 37G20, 37G40

Submitted to: Nonlinearity

\section{Introduction}

Heteroclinic orbits between saddle-like invariant sets are of great interest in dynamical systems since they generate many kinds of non-trivial behaviour, including intermittency and chaotic dynamics [1]. In continuous-time dynamical systems (i.e. ordinary differential equations) a non-transversal intersection may give rise to intermittent dynamics in the sense that trajectories approach neighbourhoods of the saddle-like invariant sets and spent a substantial amount of time there before moving rapidly to a neighbourhood of the next invariant set.

$\S$ Corresponding author (c.postlethwaite@math.auckland.ac.nz) 
It is well known that, while heteroclinic orbits in generic systems of nonlinear ordinary differential equations (ODEs) are of codimension at least one, in sets of ODEs containing invariant subspaces they can exist for open sets of parameter values, that is, they are codimension zero, and hence are referred to as 'robust' $[1,2]$. Three situations in which such invariant subspaces can arise are (i) due to equivariance with respect to a symmetry group [3, 4], (ii) modelling assumptions such as the permanence of death in Lotka-Volterra-type models of population dynamics $[5,6,7]$, and (iii) structural restrictions such as the coupled cell structures investigated recently by Aguiar et al. [8]. Detailed results on the existence of homoclinic cycles in particular families of equivariant vector fields have been given by many authors, for example $[9,10,11]$.

A number of codimension-one bifurcations have been identified in which robust heteroclinic cycles are created or destroyed, or in which their stability changes. Issues of stability turn out to be more subtle than might be at first thought. In particular, weaker definitions of stability than asymptotic stability turn out to be useful, and indeed for some systems describe the generic case. The most prevalent of these weaker notions is 'essential asymptotic stability', introduced by Melbourne [12] who gave an example of an essentially asymptotically stable robust heteroclinic cycle. Recent work by Driesse and Homburg [13] discusses examples of essentially asymptotically stable homoclinic cycles which are produced in codimension-one bifurcations from asymptotically stable robust homoclinic cycles as a 'transverse' eigenvalue (which we shall define in section 2) crosses the imaginary axis.

In this paper we discuss a particularly simple class of homoclinic cycles in $\mathbb{R}^{n}$. Although necessarily restrictive, this class contains the essence of the Lotka-Volterra type examples discussed at length by Rabinovich and co-workers $[14,15,16,17]$ as models for neural decision-making processes. The class of homoclinic cycles that we consider can undergo both transverse and resonance bifurcations and we focus here on the resonant case, beginning by proving necessary and sufficient conditions for asymptotic stability, improving on the general result given by Krupa and Melbourne [3, 4]. Such a resonance bifurcation is shown to yield a single periodic orbit which lies close to the (now unstable) homoclinic cycle. The stability and direction of bifurcation of this periodic orbit depends on coefficients in the Poincaré return map which come from the global maps, that is, those which describe how trajectories near the homoclinic cycle behave outside neighbourhoods of the equilibria on the cycle [18]. Usually these coefficients are impossible to compute analytically, but in this case the calculation turns out to be tractable, asymptotically in the limit where the sum of the transverse eigenvalues is small. To the best of our knowledge no calculation along these lines has been attempted previously. The result of the calculation is to show that the resonance bifurcation for this class of simple homoclinic cycles is always supercritical, at least when the sum of the transverse eigenvalues is sufficiently small compared to the leading expanding and contracting eigenvalues.

Field and Swift [19] study in detail a particular example from the class of cycles we consider, in the case $n=4$. They conjecture that the resonant homoclinic bifurcation 
is always supercritical. Our results prove this conjecture for an open set of parameter values.

The outline of the paper is as follows. In section 2 we introduce our notation and the class of robust homoclinic cycles that we study. We state our result on asymptotic stability of such cycles (Theorem 1). Section 3 contains the proof of Theorem 1. In section 4 we present the calculation of the coefficients in the Poincaré return map. The calculations are reasonably straightforward to follow but become remarkably lengthy. Some more detailed parts of the calculations are relegated to the Appendix. In section 5 we use our return map calculations to provide an estimate of the period of the bifurcating periodic orbit. We also show that the agreement between the analytic leading-order estimate and numerical results is very good. We conclude in section 6 .

\section{Robust homoclinic cycles}

We consider continuous time dynamical systems in the form of $\Gamma$-equivariant ODEs:

$$
\dot{x}=f(x), \quad x \in \mathbb{R}^{n}
$$

where $\Gamma \subset \mathbf{O}(n)$ is a finite Lie group. An equilibrium $\xi \in \mathbb{R}^{n}$ of (1) satisfies $f(\xi)=0$. We begin by giving a number of definitions; these are all standard in the literature, see for example $[1,4]$.

Definition $1 \phi_{j}(t)$ is a heteroclinic orbit between two equilibria $\xi_{j}$ and $\xi_{j+1}$ of (1) if $\phi_{j}(t)$ is a solution of (1) which is backward asymptotic to $\xi_{j}$ and forward asymptotic to $\xi_{j+1}$.

Definition $2 A$ heteroclinic cycle is an invariant set $X \subset \mathbb{R}^{n}$ consisting of the union of a set of equilibria $\left\{\xi_{1}, \ldots, \xi_{m}\right\}$ and orbits $\left\{\phi_{1}, \ldots, \phi_{m}\right\}$, where $\phi_{j}$ is a heteroclinic orbit between $\xi_{j}$ and $\xi_{j+1}$; and $\xi_{m+1} \equiv \xi_{1}$. We require that $m \geq 2$.

In the case $m=1$, that is, $\xi_{2}=\xi_{1}$, we say that $\phi_{1}$ is a homoclinic orbit to $\xi_{1}$.

Definition 3 A heteroclinic cycle is a homoclinic cycle if there exists $\gamma \in \Gamma$ such that $\gamma \xi_{j}=\xi_{j+1}$ for all $j$.

Definition 4 For $x \in \mathbb{R}^{n}$ the isotropy subgroup $\Sigma_{x}$ is

$$
\Sigma_{x}=\{\sigma \in \Gamma: \sigma x=x\} .
$$

Definition 5 For $\Sigma$ an isotropy subgroup of $\Lambda$, the fixed-point subspace Fix $\Sigma$ is

$$
\text { Fix } \Sigma=\left\{x \in \mathbb{R}^{n}: \sigma x=x \forall \sigma \in \Sigma\right\} \text {. }
$$

Definition 6 A heteroclinic cycle $X$ is robust if for each $j, 1 \leq j \leq m$, there exists a fixed-point subspace, $P_{j}=$ Fix $\Sigma_{j}$ where $\Sigma_{j} \subset \Lambda$ and

(i) $\xi_{j}$ is a saddle and $\xi_{j+1}$ is a sink for the flow restricted to $P_{j}$, 
Table 1. Classification of eigenvalues. $P \ominus L$ denotes the orthogonal complement in $P$ of the subspace $L$.

\begin{tabular}{ll}
\hline Eigenvalue class & Subspace \\
\hline Radial $(r)$ & $L_{j} \equiv P_{j-1} \cap P_{j}$ \\
Contracting $(c)$ & $V_{j}(c)=P_{j-1} \ominus L_{j}$ \\
Expanding $(e)$ & $V_{j}(e)=P_{j} \ominus L_{j}$ \\
Transverse $(s)$ & $V_{j}(s)=\left(P_{j-1}+P_{j}\right)^{\perp}$ \\
\hline
\end{tabular}

(ii) there is a heteroclinic connection from $\xi_{j}$ to $\xi_{j+1}$ contained in $P_{j}$.

Importantly, robust heteroclinic cycles may occur as codimension-zero phenomena in systems with symmetry. That is, they may exist for open sets of parameter values. We define $L_{j} \equiv P_{j-1} \cap P_{j}$ and clearly $\xi_{j} \in L_{j}$. Following [3], the eigenvalues of the linearisation of $f(x)$ about each equilibrium can be classified according to the subspaces in which the eigenspaces lie, as shown in table 1.

The specific differential equations we consider in this paper are of the form:

$$
\dot{x}=f(x)=\mu x+Q(x), \quad x \in \mathbb{R}^{n}
$$

where $\mu \in \mathbb{R}_{+}$(and so, following a rescaling of time, can be set equal to 1 ), $n \geq 3$, and $Q(x)$ is a $\Gamma$-equivariant polynomial, that is,

$$
\gamma Q(x)=Q(\gamma x), \quad \gamma \in \Gamma,
$$

which contains only nonlinear terms. The group $\Gamma$ is of the form

$$
\Gamma=\mathbb{Z}_{n} \ltimes \Delta_{n},
$$

where $\Delta_{n} \equiv \mathbb{Z}_{2}^{n}$ acts on $\mathbb{R}^{n}$ as $n$ reflections $\kappa_{j}, j=1, \ldots, n$ :

$$
\kappa_{j}\left(x_{1}, \ldots, x_{j}, \ldots, x_{n}\right)=\left(x_{1}, \ldots,-x_{j}, \ldots, x_{n}\right),
$$

The actions of the reflections $\kappa_{j}$ mean that every coordinate hyperplane is a fixed-point subspace, and hence invariant under (4). The $\mathbb{Z}_{n}$ subgroup of $\Gamma$ is generated by a cyclic permutation element $\rho$ which acts as:

$$
\rho\left(x_{1}, \ldots, x_{n}\right)=\left(x_{n}, x_{1}, \ldots, x_{n-1}\right) .
$$

It is clearly sufficient to consider the dynamics restricted to the domain

$$
\mathbb{R}_{+}^{n}=\left\{\left(x_{1}, \ldots, x_{n}\right) \in \mathbb{R}^{n} \mid x_{1}, \ldots, x_{n} \geq 0\right\} .
$$

We now describe the robust homoclinic cycles $X$ that we study in this paper. For now, we suppose that it is possible to construct a vector field with the following properties. In section 4 we give an explicit example of ODEs that contain such a cycle. We make the following assumptions on the ODEs (4):

(H1) There exist $n$ equilibria $\xi_{j}, j=1, \ldots n$, related by the symmetry element $\rho$ :

$$
\xi_{j+1}=\rho \xi_{j} .
$$

each with isotropy exactly $\Delta_{n-l},(1 \leq l \leq n-2)$, and therefore with $l$ non-zero coordinates. 
(H2) The unstable manifold of $\xi_{j}, W^{u}\left(\xi_{j}\right)$, is one-dimensional, and has isotropy $\Delta_{n-l-1}$, that is, points on the manifold have $(l+1)$ non-zero coordinates.

Without loss of generality, we can choose the non-zero coordinates of $\xi_{1}$ to be $x_{1}, \ldots, x_{l}$, and the non-zero coordinates of $W^{u}\left(\xi_{1}\right)$ to be $x_{1}, \ldots, x_{l+1}$. Therefore $\xi_{1}$ and $\xi_{2}$ are contained in the subspace $P_{1}$, spanned by $x_{1}, \ldots, x_{l+1}$. We make the further assumption:

(H3) $\xi_{2}$ is the only sink in $P_{1}$ and the unstable manifold of $\xi_{1}$ is asymptotic to $\xi_{2}$.

Therefore the union of the $\xi_{j}$ and their unstable manifolds forms a homoclinic cycle we label $X$.

From the action of $\rho^{-1}$, it is clear that $P_{n}$ is spanned by $x_{n}, x_{1}, \ldots, x_{l}$. Using table 1 we deduce that $V_{1}(c)$ is the $x_{n}$ axis and $V_{1}(e)$ is the $x_{l+1}$ axis. The radial subspace of $\xi_{1}$ has $l$ dimensions, and so there are $n-l-2$ transverse directions. Since $\xi_{j}$ has a onedimensional unstable manifold, all the transverse and radial eigenvalues are negative.

We label the contracting eigenvalue (that is, the eigenvalue in the direction spanned by $V_{j}(c)$ at $\left.\xi_{j}\right)-c<0$, and the expanding eigenvalue (the eigenvalue in the direction spanned by $\left.V_{j}(e)\right), e>0$. We label the transverse eigenvalues $-s_{1}, \ldots,-s_{n-l-2}$ where the eigenvector corresponding to the eigenvalue $-s_{k}$ at $\xi_{j}$ is in the $x_{l+j+k}$ direction, taking subscripts modulo $n$ as appropriate.

Theorem 1 The homoclinic cycle $X$ is asymptotically stable if

$$
c+\sum_{j=1}^{n-l-2} s_{j}>e .
$$

If $c+\sum_{j=1}^{n-l-2} s_{j}<e$ then the cycle is unstable.

When the stability of the cycle changes at $c+\sum_{j=1}^{n-l-2} s_{j}=e$, the cycle undergoes a resonance bifurcation. In this bifurcation a unique long-period periodic orbit bifurcates; the bifurcation may be supercritical (in which case the bifurcating periodic orbit is stable and exists for parameters for which $X$ is unstable) or subcritical (and the bifurcating periodic orbit is unstable and exists for parameters for which $X$ is stable). It turns out that the criticality of the resonance bifurcation is determined by the behaviour of trajectories in the 'global parts' of the flow near $X$ (i.e. the parts of the flow away from neighbourhoods of the equilibria). In the second half of this paper we give an example of a system which contains a homoclinic cycle of the form described above, and we explicitly calculate these constants. For the example we give, we show that the bifurcation always occurs supercritically. The calculation also allows us to compute the period of the bifurcating stable periodic orbit.

\section{Proof of theorem 1}

This section is devoted to the proof of theorem 1. The proof is divided into three sections; first we construct return maps on Poincaré sections in the standard way by 
dividing the flow into 'local' and 'global' parts. The return maps describe the behaviour of trajectories asymptotically close to $X$. We then relate these maps to transition matrices, and finally deduce results on the eigenvalues of the transition matrices which enable us to prove the theorem.

We remark that, although this result can be deduced from a theorem of Hofbauer and Sigmund [7] (see chapter 17, pages 220-232), we see substantial value in the proof presented here since it is more transparent for the situation at hand, and it makes the geometry of the invariant subspaces explicit.

\subsection{Poincaré maps}

We define Poincaré intersections about $\xi_{1}$ in the standard way:

$$
H_{1}^{\text {in }}:\left\{x_{n}=h^{2}\right\}, \quad H_{1}^{\text {out }}:\left\{x_{l+1}=h^{2}\right\},
$$

for some small $h>0$. We consider an initial point on $H_{1}^{\text {in }}$ :

$$
x^{\mathrm{in}, 1}=\left(v_{1}^{\mathrm{in}, 1}, \ldots, v_{l}^{\mathrm{in}, 1}, x_{l+1}^{\mathrm{in}, 1}, \ldots, x_{n-1}^{\mathrm{in}, 1}, h^{2}\right),
$$

where the $v_{j}$ are radial coordinates which are zero at $\xi_{1}$. The 'time of flight' for the trajectory to reach $H_{1}^{\text {out }}$ is

$$
T_{1}=-\frac{1}{e} \log \left(\frac{x_{l+1}^{\mathrm{in}, 1}}{h^{2}}\right) .
$$

Let the coordinates of the trajectory when it reaches $H_{1}^{\text {out }}$ be

$$
x^{\text {out }, 1}=\left(v_{1}^{\text {out }, 1}, \ldots, v_{l}^{\text {out }, 1}, x_{l+1}^{\text {out }, 1}, \ldots, x_{n}^{\text {out }, 1}\right)
$$

then using the linearised flow at $\xi_{j}$, we have

$$
\begin{aligned}
& v_{j}^{\text {out }, 1}=h^{-\frac{2 r_{j}}{e}} v_{j}^{\text {in }, 1}\left(x_{l+1}^{\text {in }, 1}\right)^{\frac{r_{j}}{e}}, \quad j=1, \ldots, l \\
& x_{l+1}^{\text {out }, 1}=h^{2}, \\
& x_{j}^{\text {out }, 1}=h^{\frac{-2 s_{j-l-1}}{e}} x_{j}^{\text {in }, 1}\left(x_{l+1}^{\text {in }, 1}\right)^{\frac{s_{j-l-1}}{e}}, j=l+2, \ldots, n-1 \\
& x_{n}^{\text {out }, 1}=h^{2-\frac{2 c}{e}}\left(x_{l+1}^{\text {in }, 1}\right)^{\frac{c}{e}} .
\end{aligned}
$$

This defines the local map near $\xi_{1}$.

We now compute the global part of the Poincaré map. The isotypic decomposition of $\mathbb{R}^{n}$ with respect to the isotropy of $W^{u}\left(\xi_{1}\right)$ is

$$
\mathbb{R}^{n}=P_{1} \oplus V_{1}\left(s_{1}\right) \oplus \ldots \oplus V_{1}\left(s_{n-l-2}\right) \oplus V_{1}(c)
$$

where $V_{1}\left(s_{j}\right)=\left\langle\left(0, \ldots, 0, x_{l+j+1}, 0, \ldots, 0\right)\right\rangle$ is the eigenspace spanned by the eigenvalue $s_{j}$. Recall that $\operatorname{dim} P_{1}=l+1$. The form of the global map from $H_{1}^{\text {out }}$ to a point $x^{\text {in, } 2}$ 
on $H_{2}^{\text {in }}:\left\{x_{1}=h^{2}\right\}$, up to linear terms, is therefore:

$$
\left(\begin{array}{c}
x_{1}^{\text {in, }, 2} \\
v_{2}^{\text {in, }} \\
\vdots \\
v_{l+1}^{\text {in, }} \\
x_{l+2}^{\text {in, }} \\
\vdots \\
x_{n}^{\text {in, }}
\end{array}\right)=\left(\begin{array}{c}
h^{2} \\
w_{2}^{\star} \\
\vdots \\
w_{l+1}{ }^{\star} \\
0 \\
\vdots \\
0
\end{array}\right)+\left(\begin{array}{ccccccc}
0 & \cdots & 0 & 0 & 0 & \cdots & 0 \\
c_{21} & \cdots & c_{2 l} & c_{2 l+1} & 0 & \cdots & 0 \\
\vdots & & \vdots & \vdots & \vdots & & \vdots \\
c_{l+11} & \cdots & c_{l+1 l} & c_{l+1 l+1} & 0 & \cdots & 0 \\
0 & \cdots & 0 & 0 & c_{l+2 l+2} & \cdots & 0 \\
\vdots & & \vdots & \vdots & \vdots & \ddots & \vdots \\
0 & \cdots & 0 & 0 & 0 & \cdots & c_{m m}
\end{array}\right)\left(\begin{array}{c}
v_{1}^{\text {out }, 1} \\
\vdots \\
\vdots \\
v_{l}^{\text {out }, 1} \\
h^{2} \\
x_{l+2}^{\text {out }, 1} \\
\vdots \\
x_{n}^{\text {out }, 1}
\end{array}\right)
$$

where the $c_{i j}$ are $O(1)$ constants which depend on the global flow. By the invariance of the coordinate planes, $c_{j j}>0$ for $l+2 \leq j \leq n$. In the section 4 , we find approximations for these $c_{j j}$ for a specific set of ODEs. The $w_{j}{ }^{\star}$ are $O\left(h^{2}\right)$ constants which define where the heteroclinic connection $W^{u}\left(\xi_{1}\right)$ hits the plane $H_{2}^{\text {in }}$. Note that $x_{1}^{\text {in, } 2}=h^{2}$ by definition, and the radial coordinates on $H_{2}^{\text {in }}$ are $v_{2}^{\text {in, }, 2}, \ldots, v_{l+1}^{\text {in, } 2}$.

We compose the local and global maps, and use the symmetry $\rho^{-1}$ to map points on $H_{2}^{\text {in }}$ onto $H_{1}^{\text {in }}$, to give a return map $\phi$ on $H_{1}^{\text {in }}$. That is, we associate the point $\rho^{-1} x^{\text {in, } 2}$ with a point on $H_{1}^{\text {in }}$, so

$$
\phi\left(x^{\mathrm{in}, 1}\right)=\rho^{-1}\left(x^{\mathrm{in}, 2}\right),
$$

or

$$
\phi\left(\begin{array}{c}
v_{1}^{\text {in,1 }} \\
\vdots \\
v_{l}^{\text {in, }, 1} \\
x_{l+1}^{\text {in, }} \\
x_{l+2}^{\text {in, }} \\
\vdots \\
x_{n}^{\text {in, },}
\end{array}\right)=\rho^{-1}\left(\begin{array}{c}
x_{1}^{\text {in, }, 2} \\
v_{2}^{\text {in,2 }} \\
\vdots \\
v_{l+1}^{\text {in,2 }} \\
x_{l+2}^{\text {in,2 }} \\
\vdots \\
x_{n}^{\text {in, }, 2}
\end{array}\right)=\left(\begin{array}{c}
v_{2}^{\text {in, }, 2} \\
\vdots \\
v_{l+1}^{\text {in, }} \\
x_{l+2}^{\text {in, }} \\
\vdots \\
x_{n}^{\text {in, }, 2} \\
x_{1}^{\text {in, }, 2}
\end{array}\right) .
$$

The radial $\left(v_{j}\right)$ components are contracting, and do not affect the other components, so we only need consider an $n-l-1$ dimensional map $\psi$ that describes the dynamics of the flow near the homoclinic cycle:

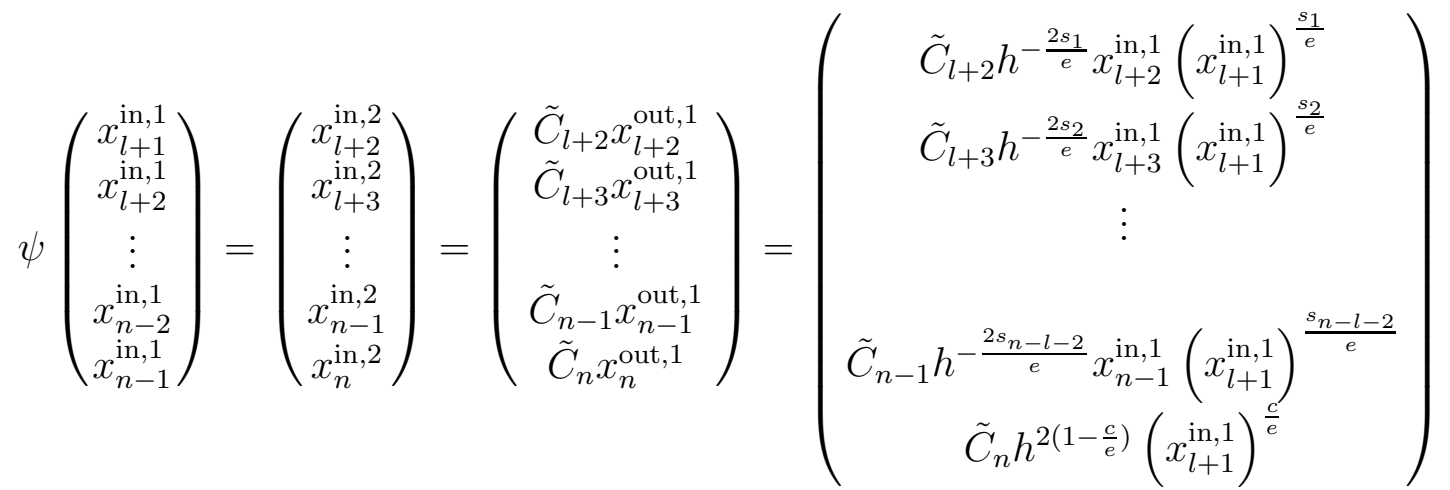

where $\tilde{C}_{j}=c_{j j}$. For convenience, we relabel the coordinates and constants. We write $p=n-l-1$,

$$
y_{j}=x_{l+j}, j=1, \ldots, p,
$$


Resonance bifurcations from robust homoclinic cycles

$$
C_{j}=h^{-\frac{2 s_{j}}{e}} \tilde{C}_{l+j}, \text { for } 1 \leq j \leq p-1, \quad C_{p}=h^{2\left(1-\frac{c}{e}\right)} \tilde{C}_{n},
$$

and

$$
a_{j}=\frac{s_{j}}{e}, \text { for } 1 \leq j \leq p-1, \quad a_{p}=\frac{c}{e}
$$

to reach:

$$
\psi\left(\begin{array}{c}
y_{1} \\
\vdots \\
y_{p-1} \\
y_{p}
\end{array}\right)=\left(\begin{array}{c}
C_{1} y_{2} y_{1}^{a_{1}} \\
\vdots \\
C_{p-1} y_{p} y_{1}^{a_{p-1}} \\
C_{p} y_{1}^{a_{p}}
\end{array}\right)
$$

The map $\psi$ has a fixed point at $y_{1}=\ldots=y_{p}=0$ which corresponds to the homoclinic cycle in the flow. The map has a second fixed point, the $y_{1}$ coordinate of which satisfies

$$
y_{1}=\left(\prod_{j=1}^{p} C_{j}\right)\left(y_{1}\right)^{\delta},
$$

where

$$
\delta=\sum_{j=1}^{p} a_{j}=\frac{c+\sum_{j=1}^{p-1} s_{j}}{e} .
$$

Note that $\prod_{j=1}^{p} C_{j}=h^{2(1-\delta)} \prod_{j=l+1}^{n} \tilde{C}_{j}$. This fixed point may correspond to a periodic orbit in the flow, and we discuss the existence and stability of this fixed point further in section 4 below.

In the next section we use transition matrix methods to determine the stability of the zero fixed point of $\psi$ and hence the stability of the homoclinic cycle in the flow.

\subsection{Transition matrices}

Let $G$ be the set of mappings $g: \mathbb{R}^{p} \rightarrow \mathbb{R}^{p}$ that have at lowest order the form

$$
g\left(x_{1}, \ldots, x_{p}\right)=\left(C_{1} x_{1}^{\alpha_{11}} x_{2}^{\alpha_{12}} \cdots x_{p}^{\alpha_{1 p}}, \ldots, C_{p} x_{1}^{\alpha_{p 1}} \cdots x_{p}^{\alpha_{p p}}\right)
$$

for constants $\alpha_{i j} \geq 0$ and $C_{i}$ non-zero. $G$ is clearly closed under composition. We define the transition matrix [3, 19] of $g$ to be the $p \times p$ real matrix $M(g)$ with entries $[M(g)]_{i j}=\alpha_{i j}$. It is easily verified that if $g_{1}, g_{2} \in G$, then

$$
M\left(g_{2} \circ g_{1}\right)=M\left(g_{2}\right) M\left(g_{1}\right) .
$$

Any $g \in G$ has a fixed point at $x_{1}=\ldots=x_{p}=0$. The zero fixed point of the map $g$ will be stable if all the row sums of $M(g)^{N}$ diverge to $+\infty$ as $N \rightarrow \infty$. Conversely, if any of the row sums of $M(g)^{N}$ tends to 0 , then the fixed point is unstable. 
For the homoclinic cycles described above, the transition matrix corresponding to the Poincaré map (14) is:

$$
M(\psi)=A_{p}=\left(\begin{array}{ccccc}
a_{1} & 1 & 0 & \cdots & 0 \\
a_{2} & 0 & 1 & \cdots & 0 \\
\vdots & \vdots & \ddots & \ddots & \vdots \\
a_{p-1} & 0 & \cdots & 0 & 1 \\
a_{p} & 0 & \cdots & 0 & 0
\end{array}\right)
$$

with the $a_{i}$ as in (13). We now recall the Perron-Frobenius theorem. We write $M \cdot \mathbf{v}$ to be the product $M_{i j} v_{j}$ for a matrix $M$ with components $M_{i j}$ and vector $\mathbf{v}$ with components $v_{j}$. The row sums of $A_{p}^{N}$ can be written as $A_{p}^{N} \cdot \mathbf{1}$ where $\mathbf{1}$ is the vector $(1,1, \ldots, 1)^{T}$.

Definition 7 A real $p \times p$ matrix $M$ is primitive if there exists an $N>0$ such that all entries of $M^{N}$ are strictly positive.

By inspection, $A_{p}$ is primitive, since $a_{i}>0$ for all $i$, and so $\left(A_{p}\right)^{p}$ will have strictly positive entries.

Theorem 2 (Perron-Frobenius) If $M$ is a $p \times p$ non-negative primitive matrix, then there exists a unique and simple positive eigenvalue $\lambda_{\mathrm{pf}}$ which is dominant in the sense that $|\lambda|<\lambda_{\mathrm{pf}}$ for all other eigenvalues $\lambda$ of $M$. There exist right and left eigenvectors $\mathbf{u}, \mathbf{v}>\mathbf{0}$ such that $M \cdot \mathbf{u}=\lambda_{\mathrm{pf}} \mathbf{u}$ and $\mathbf{v}^{\top} \cdot M=\lambda_{\mathrm{pf}} \mathbf{v}^{\top}$. If $\mathbf{u}$ and $\mathbf{v}$ are normalised such that $\mathbf{v}^{\top} \cdot \mathbf{u}=1$ then

$$
\lim _{N \rightarrow \infty}\left(\lambda_{\mathrm{pf}}^{-N} M^{N}\right)=T \equiv \mathbf{u v}^{\top} .
$$

We now consider matrices of the form $A_{p}$, with $a_{i}>0$, and in the remainder of this section, prove the following lemma.

Lemma 1 If $\sum_{j=1}^{p} a_{j}<1$, then the dominant eigenvalue $\lambda_{\mathrm{pf}}$ of $A_{p}$ satisfies $\lambda_{\mathrm{pf}}<1$. If $\sum_{j=1}^{p} a_{j}>1$ then $\lambda_{\mathrm{pf}}>1$.

Using the Perron-Frobenius theorem, we have

$$
\lim _{N \rightarrow \infty} \lambda_{\mathrm{pf}}^{-N} A_{p}^{N} \cdot \mathbf{1}=T \cdot \mathbf{1}=\mathbf{u}^{\prime}>\mathbf{0},
$$

where $\mathbf{u}^{\prime}=\left(\sum_{j} v_{j}\right) \mathbf{u}$. If $\lambda_{\mathrm{pf}}<1$, then the row sums tend to zero, that is, $\lim _{N \rightarrow \infty} A_{p}^{N} \cdot \mathbf{1}=0$, and so the zero fixed point of the map $\psi$ is unstable. If $\lambda_{\mathrm{pf}}>1$, then $A_{p}^{N} \cdot 1$ will thus be divergent to $+\infty$, and the zero fixed point of the map will be stable. Since the stability of the fixed point in the map corresponds to the stability of the homoclinic cycle in the flow, substituting for the $a_{j}$ from (13) completes the proof of theorem 1.

We now prove lemma 1.

Claim 1 The eigenvalues $\lambda$ of $A_{p}$ satisfy

$$
\lambda^{p}-\left(a_{1} \lambda^{p-1}+a_{2} \lambda^{p-2}+\ldots+a_{p-1} \lambda+a_{p}\right)=0 .
$$


Proof. We will show by induction (on $p$ ) that

$D_{p} \equiv \operatorname{det}\left(A_{p}-\lambda I\right)=(-1)^{p}\left[\lambda^{p}-\left(a_{1} \lambda^{p-1}+a_{2} \lambda^{p-2}+\ldots+a_{p-1} \lambda+a_{p}\right)\right]$.

Firstly, we have

$$
D_{1}=a_{1}-\lambda=(-1)\left[\lambda-a_{1}\right] .
$$

Then

$$
D_{k}=\left|\begin{array}{ccccc}
a_{1}-\lambda & 1 & 0 & \cdots & 0 \\
a_{2} & -\lambda & 1 & \cdots & 0 \\
\vdots & \vdots & \ddots & \ddots & \vdots \\
a_{k-1} & 0 & \cdots & -\lambda & 1 \\
a_{k} & 0 & \cdots & 0 & -\lambda
\end{array}\right|=-\lambda D_{k-1}+(-1)^{k-1} a_{k} .
$$

Assuming (16) for $p=k-1$ we have

$$
\begin{gathered}
D_{k+1}=-\lambda(-1)^{k}\left[\lambda^{k}-\left(a_{1} \lambda^{k-1}+a_{2} \lambda^{k-2}+\ldots+a_{k-1} \lambda+a_{k}\right)\right]+(-1)^{k} a_{k+1} \\
=(-1)^{k+1}\left[\lambda^{k+1}-\left(a_{1} \lambda^{k}+a_{2} \lambda^{k-1}+\ldots+a_{k} \lambda+a_{k+1}\right)\right] .
\end{gathered}
$$

Hence (16) is satisfied for all $p \geq 1$.

The roots of equation (15) are the eigenvalues of $A_{p}$. The Perron-Frobenius theorem states that there is at least one positive root, the next claim relates the size of the positive $\operatorname{root}(\mathrm{s})$ to the entries $a_{j}$ of $A_{p}$.

Claim 2 Let $\lambda$ be a positive root of (15). If $\sum_{j=1}^{p} a_{j}<1$ then $\lambda<1$; if $\sum_{j=1}^{p} a_{j}>1$ then $\lambda>1$.

Proof. Consider the case $\sum_{j=1}^{p} a_{j}<1$, and suppose that $\lambda \geq 1$ :

$$
\lambda^{p}=a_{1} \lambda^{p-1}+a_{2} \lambda^{p-2}+\ldots+a_{p-1} \lambda+a_{p} \leq \lambda^{p-1} \sum_{j=1}^{p} a_{j}<\lambda^{p-1} \leq \lambda^{p} .
$$

This is a contradiction and hence $\lambda<1$. Similarly, for the case $\sum_{j=1}^{p} a_{j}>1$, suppose that $\lambda \leq 1$, and then

$$
\lambda^{p}=a_{1} \lambda^{p-1}+a_{2} \lambda^{p-2}+\ldots+a_{p-1} \lambda+a_{p} \geq \lambda^{p-1} \sum_{j=1}^{p} a_{j}>\lambda^{p-1} \geq \lambda^{p},
$$

again a contradiction, and hence $\lambda>1$.

Claim 2 is true for all positive roots $\lambda$, therefore it is certainly true for the dominant eigenvalue $\lambda_{\mathrm{pf}}$, completing the proof of lemma 1.

\section{Bifurcation criticality computation}

In this section we give an example set of ODEs which contain a homoclinic cycle of the type discussed in section 2 . For these equations, we are able to explicitly compute the shape of the heteroclinic connections between the equilibria, and use this to calculate the

unknown constants $\tilde{C}_{j}$ in the global part of the Poincaré map (12) derived in section 3.1. This allows us to compute the criticality of the resonance bifurcation and in addition, the period of the resulting bifurcating orbit. 


\subsection{System description}

The following equations satisfy conditions (H1) - (H3) and are equivariant under the action of $\Gamma$ given in (5), (6) and (7).

$\dot{x}_{j}=x_{j}\left(1-\sum_{i=1}^{n} x_{i}^{2}-c x_{j+1}^{2}-s_{n-3} x_{j+2}^{2}-\cdots-s_{1} x_{j-2}^{2}+e x_{j-1}^{2}\right), \quad j=1, \ldots, n$

for $x=\left(x_{1}, \ldots, x_{n}\right) \in \mathbb{R}^{n}, c, e, s_{j}>0,(j=1, \ldots, n-3)$. In addition, in this example, $l=1$, and $Q(x)$ is truncated at third order. We consider only $n \geq 4$. The case $n=3$ is the example of Guckenheimer and Holmes [11] and it is well known that the system truncated at third order has a degenerate resonance bifurcation at $c=e$.

The system (17) has $n$ equilibria on the coordinate axes we label $\xi_{j}$; the equilibrium $\xi_{j}$ has coordinates $x_{j}=1, x_{k}=0, k \neq j$. Each equilibrium is hyperbolic, and the eigenvalues of the linearisation about $\xi_{j}$ are -2 (in the radial, that is, $x_{j}$ direction), $-c$ (in the $x_{j-1}$ direction), $e$ (in $x_{j+1}$ direction), and $-s_{k}$ in the $x_{k+j+1}$ direction $(k=1, \ldots, n-3)$. Note that a homoclinic cycle between the equilibria $\xi_{j}$ exists if $-c$ and $e$ are of opposite sign, but without loss of generality we consider only the case $c, e>0$. Thus $e$ is the expanding eigenvalue and $c$ is the contracting eigenvalue. The $-s_{j}$ are transverse eigenvalues; we assume $-s_{j}<0$. By theorem 1 , the homoclinic cycle is asymptotically stable if $c+\sum_{j=1}^{n-3} s_{j}>e$. Otherwise it is unstable.

In section 3.1 we constructed a Poincaré map (12) on a section $H_{1}^{\text {in }}$, approximating the flow near the homoclinic cycle. This map has a non-trivial fixed point with $x_{2^{-}}$ coordinate given by

$$
x_{2}=h^{2}\left(\prod_{j=l+2}^{n} \tilde{C}_{j}\right)^{\frac{1}{1-\delta}}
$$

where the $\tilde{C}_{j}$ are defined in the map (12).

This point corresponds to a periodic orbit in the flow, branching from the resonant

homoclinic bifurcation, only if it is small as $\delta \rightarrow 1$. Let $C=\left(\prod_{j=l+2}^{n} \tilde{C}_{j}\right)$. For $C<1$ the fixed point is small if $\delta<1$ (where the heteroclinic cycle is unstable), so the bifurcation is supercritical. Conversely for $C>1$ the orbit exists in $\delta>1$, and the bifurcation is subcritical. In the following, we write $A=\log C$. Thus the bifurcation is supercritical if $A<0$.

\subsection{The solution for the heteroclinic trajectory}

We now compute an approximation for the form of the heteroclinic trajectory near the resonance bifurcation, under the assumption that the difference between the expanding and contracting eigenvalues, $e-c$, is small. We write $e=c+\beta$ and take $|\beta| \ll 1$. We consider the heteroclinic connection from $\xi_{1}$ to $\xi_{2}$ in the $x_{1}-x_{2}$ plane; all other connections are symmetry-related. The equations in the $x_{1}-x_{2}$ plane are:

$$
\begin{aligned}
& \dot{x}_{1}=x_{1}\left(1-\left(x_{1}^{2}+x_{2}^{2}\right)-c x_{2}^{2}\right), \\
& \dot{x}_{2}=x_{2}\left(1-\left(x_{1}^{2}+x_{2}^{2}\right)+e x_{1}^{2}\right) .
\end{aligned}
$$


For ease of computation, we make a change of variables, writing $u=x_{1}^{2}, v=x_{2}^{2}$ :

$$
\begin{aligned}
& \dot{u}=2 u(1-(u+v)-c v) \\
& \dot{v}=2 v(1-(u+v)+e u)
\end{aligned}
$$

The heteroclinic connection is a solution to (19) and (20) with boundary conditions $\{u=0, v=1\}$ and $\{v=0, u=1\}$. When $c=e(\beta=0)$, there exists an exact solution $v=v_{0}(u) \equiv 1-u$.

Recall that at resonance,

$$
c+s=e,
$$

where $s=\sum_{j=1}^{n-3} s_{j}$. Since $e=c+\beta$, at resonance we also have

$$
s=\beta .
$$

Therefore, close to resonance, $s$ is $O(\beta)$, although it is important to note that it is not necessary that each $s_{j}$ is individually $O(\beta)$. We will now look for approximate solutions to (19) and (20) when $\beta \neq 0$. Write

$$
v=v_{0}(u)+\beta v_{1}(u)+O\left(\beta^{2}\right) .
$$

Expanding equations (19) and (20) in powers of $\beta$, we find at $O(\beta)$ :

which gives

$$
v_{1}^{\prime}(u)-\frac{1}{c u(1-u)} v_{1}(u)=-\frac{1}{c},
$$

$$
v_{1}(u)=\frac{1}{c}\left(\frac{u}{1-u}\right)^{1 / c} \int_{u}^{1}\left(\frac{1-\xi}{\xi}\right)^{1 / c} \mathrm{~d} \xi .
$$

In the following we also find it convenient to write the heteroclinic connection as a function $u(v)$, that is, we write the connection in the form

$$
u(v)=1-v+\beta u_{1}(v)+O\left(\beta^{2}\right) .
$$

By rearranging (23) and substituting into (21) we find that $u_{1}(v)=v_{1}(1-v)$, that is

$$
u_{1}(v)=\frac{1}{c}\left(\frac{1-v}{v}\right)^{1 / c} \int_{0}^{v}\left(\frac{\eta}{1-\eta}\right)^{1 / c} \mathrm{~d} \eta .
$$

\subsection{Constants in the global map}

We now use the approximated form of the homoclinic connection (21) to compute the constants $\tilde{C}_{j}$ in the map (12). From (12) we have

$$
\tilde{C}_{j}=\frac{x_{j}^{\text {in, }, 2}}{x_{j}^{\text {out }, 1}}, \quad j=3, \ldots, n .
$$

Consider a trajectory close to the $x_{1}-x_{2}$ plane, so that all other coordinates are small. Approximations to the $x_{3^{-}}, \ldots,-x_{n}$ equations in (17) are therefore (ignoring higher order terms in the small coordinates):

$$
\begin{aligned}
& \dot{x}_{3}=x_{3}\left(1-\left(x_{1}^{2}+x_{2}^{2}\right)-s_{1} x_{1}^{2}+e x_{2}^{2}\right), \\
& \dot{x}_{j}=x_{j}\left(1-\left(x_{1}^{2}+x_{2}^{2}\right)-s_{j-2} x_{1}^{2}-s_{j-3} x_{2}^{2}\right), \quad j=4, \ldots, n-1, \\
& \dot{x}_{n}=x_{n}\left(1-\left(x_{1}^{2}+x_{2}^{2}\right)-c x_{1}^{2}-s_{n-3} x_{2}^{2}\right) .
\end{aligned}
$$


We consider a trajectory which starts close to the heteroclinic cycle. This trajectory will approximately follow the heteroclinic trajectory in the $x_{1}-x_{2}$ plane, so we integrate along the trajectory, from $H_{1}^{\text {out }}$ to $H_{2}^{\text {in }}$. So from (26) for instance, we find:

$$
\int_{H_{1}^{\text {out }}}^{H_{2}^{\text {in }}} \frac{1}{x_{3}} \mathrm{~d} x_{3}=\int_{H_{1}^{\text {out }}}^{H_{2}^{\text {in }}}\left(1-\left(x_{1}^{2}+x_{2}^{2}\right)-s_{1} x_{1}^{2}+e x_{2}^{2}\right) \mathrm{d} t .
$$

We can compute the left hand side of (29):

$$
\int_{H_{1}^{\text {out }}}^{H_{2}^{\text {in }}} \frac{1}{x_{3}} \mathrm{~d} x_{3}=\log \left(x_{3}^{\text {in }, 2}\right)-\log \left(x_{3}^{\text {out }, 1}\right)=\log \left(\frac{x_{3}^{\text {in }, 2}}{x_{3}^{\text {out }, 1}}\right)=\log \left(\tilde{C}_{3}\right),
$$

(where the last equality follows from (25)), and we find similar expressions for the other $\log \left(\tilde{C}_{j}\right)$ by integrating equations $(27)$ and $(28)$.

As noted at the end of section 4.1, the criticality of the bifurcation depends on the sign of $A$, where

$$
A=\log C=\log \left(\prod_{j=3}^{n} \tilde{C}_{j}\right)=\sum_{j=3}^{n} \log \tilde{C}_{j} .
$$

Summing the integrated forms of equations (26) to (28) we find, again writing $x_{1}^{2}=u$, and $x_{2}^{2}=v$,

$$
A=\sum_{j=3}^{n} \log \tilde{C}_{j}=\int_{H_{1}^{\text {out }}}^{H_{2}^{\text {in }}}\left((n-2)(1-(u+v))-c u-\sum_{j=1}^{n-3} s_{j}(u+v)+e v\right) \mathrm{d} t
$$

where again the integral is taken along the heteroclinic connection in the $x_{1}-x_{2}$ plane. Note that this expression does not depend independently on the $s_{j}$; it only depends on the sum $s=\sum_{j=1}^{n-3} s_{j}$.

We now rewrite the integral as an integral in $u$. Recall that the heteroclinic connection can be written as $v(u)=1-u+\beta v_{1}(u)+O\left(\beta^{2}\right)$, and $e=c+\beta$. From (19), we have

$$
\dot{u}=-2 c u(1-u)-2 \beta(c+1) u v_{1}(u)+O\left(\beta^{2}\right)
$$

SO

$$
\frac{1}{\dot{u}}=\frac{-1}{2 c u(1-u)}+\beta \frac{c+1}{2 c^{2}} \frac{v_{1}}{u(1-u)^{2}}+O\left(\beta^{2}\right) .
$$

To determine the bounds on the integral, note that on $H_{1}^{\text {out }}, x_{2}^{2}=h^{2}$, so $v=h$, and the $u$-coordinate of the heteroclinic connection is $u=1-h+\beta u_{1}(h)+O\left(\beta^{2}\right)$. On $H_{2}^{\text {in }}$, $u=h$.

Thus, expanding in powers of $\beta$ (recall that $s=O(\beta)$ ) we find:

$$
\begin{aligned}
A= & \int_{H_{1}^{\text {out }}}^{H_{2}^{\text {in }}}((n-2)(1-(u+v(u)))-c u-s(u+v(u))+e v) \frac{1}{\dot{u}} \mathrm{~d} u \\
= & \int_{1-h+\beta u_{1}(h)}^{h}\left\{-c u+c(1-u)-s+\beta\left[-(n-2-c) v_{1}(u)+(1-u)\right]\right\} \\
& \times\left\{-\frac{1}{2 c u(1-u)}+\beta\left[\frac{c+1}{2 c^{2}} \frac{v_{1}}{u(1-u)^{2}}\right]\right\} \mathrm{d} u+O\left(\beta^{2}\right),
\end{aligned}
$$


which, after rearranging terms and tidying up, becomes

$$
\begin{aligned}
2 A=\int_{1-h+\beta u_{1}(h)}^{h} & \frac{u-(1-u)}{u(1-u)} \mathrm{d} u-\beta \int_{1-h}^{h} \frac{1}{c u} \mathrm{~d} u+s \int_{1-h}^{h} \frac{1}{c u(1-u)} \mathrm{d} u \\
& +\beta \int_{1-h}^{h} \frac{(n-2-c) v_{1}(u)}{c u(1-u)}+(1-2 u) \frac{c+1}{c} \frac{v_{1}(u)}{u(1-u)^{2}} \mathrm{~d} u+O\left(\beta^{2}\right), \\
= & \int_{1-h+\beta u_{1}(h)}^{h} \frac{1}{1-u}-\frac{1}{u} \mathrm{~d} u-\frac{\beta}{c} \int_{1-h}^{h} \frac{1}{u} \mathrm{~d} u+\frac{s}{c} \int_{1-h}^{h} \frac{1}{u}+\frac{1}{1-u} \mathrm{~d} u \\
& +\frac{\beta}{c} \int_{1-h}^{h} \frac{(n-2-c)(1-u)+(1-2 u)(c+1)}{u(1-u)^{2}} v_{1}(u) \mathrm{d} u+O\left(\beta^{2}\right), \\
= & \log \left(1-\frac{\beta u_{1}(h)}{h}\right)+\log \left(1+\frac{\beta u_{1}(h)}{1-h}\right)+\frac{2 s-\beta}{c}(\log h-\log (1-h)) \\
& -\beta \frac{n-1}{c} \int_{h}^{1-h} \frac{v_{1}(u)}{u(1-u)} \mathrm{d} u+\beta \frac{c+1}{c} \int_{h}^{1-h} \frac{v_{1}(u)}{(1-u)^{2}} \mathrm{~d} u+O\left(\beta^{2}\right) .
\end{aligned}
$$

Since the functions $u_{1}$ and $v_{1}$ are (in principal) known through the integrals (24) and (22) the above expression determines the sign of $A$, and hence yields the criticality of the

resonance bifurcation. The remainder of this section is devoted to the computation of $A$ which is perhaps surprisingly algebraically complicated.

\subsection{Expansion of integrals}

There are two types of terms in $A$ which we cannot yet express explicitly: the terms involving $u_{1}(h)$ and the integrals involving $v_{1}(u)$. We will compute both of these by calculating a power series expansion for $u_{1}$. The two integrals in $A$ which we need to compute are:

$$
\int_{h}^{1-h} \frac{v_{1}(u)}{u(1-u)} \mathrm{d} u \equiv \int_{h}^{1-h} \frac{u_{1}(w)}{w(1-w)} \mathrm{d} w
$$

and

$$
\int_{h}^{1-h} \frac{v_{1}(u)}{(1-u)^{2}} \mathrm{~d} u \equiv \int_{h}^{1-h} \frac{u_{1}(w)}{w^{2}} \mathrm{~d} w .
$$

For convenience, we write $q=1 / c$, then

$$
u_{1}(w)=q\left(\frac{1-w}{w}\right)^{q} \int_{0}^{w}\left(\frac{\xi}{1-\xi}\right)^{q} \mathrm{~d} \xi .
$$

In the integrals (31) and (32) we only need to know $u_{1}(w)$ for $w<1$, so we can write the factors $(1-\xi)^{-q}$ and $(1-w)^{q}$ as power series. In the following, $\left(\begin{array}{l}q \\ k\end{array}\right)$ is a generalised binomial coefficient, that is,

$$
\left(\begin{array}{l}
q \\
k
\end{array}\right) \equiv \frac{\Gamma(q+1)}{\Gamma(k+1) \Gamma(q-k+1)}
$$

where $\Gamma(q)$ is the usual Gamma function. Substituting the expansions into $u_{1}(w)$ gives:

$$
u_{1}(w)=q w^{-q} \sum_{k=0}^{\infty}\left(\begin{array}{l}
q \\
k
\end{array}\right)(-w)^{k} \int_{0}^{w} \xi^{q} \sum_{k=0}^{\infty}\left(\begin{array}{c}
-q \\
k
\end{array}\right)(-\xi)^{k} \mathrm{~d} \xi
$$


Resonance bifurcations from robust homoclinic cycles

$$
\begin{aligned}
& =q w^{-q}\left(\sum_{k=0}^{\infty}(-1)^{k}\left(\begin{array}{l}
q \\
k
\end{array}\right) w^{k}\right)\left(\sum_{k=0}^{\infty}(-1)^{k}\left(\begin{array}{c}
-q \\
k
\end{array}\right) \int_{0}^{w} \xi^{q+k} \mathrm{~d} \xi\right) \\
& =q w^{-q}\left(\sum_{k=0}^{\infty}(-1)^{k}\left(\begin{array}{l}
q \\
k
\end{array}\right) w^{k}\right)\left(\sum_{k=0}^{\infty}(-1)^{k}\left(\begin{array}{c}
-q \\
k
\end{array}\right) \frac{1}{q+k+1} w^{q+k+1}\right) \\
& =q w \sum_{n=0}^{\infty} c_{n} w^{n}
\end{aligned}
$$

where

$$
\begin{aligned}
c_{n} & =\sum_{k=0}^{n}(-1)^{n-k}\left(\begin{array}{c}
q \\
n-k
\end{array}\right)(-1)^{k}\left(\begin{array}{c}
-q \\
k
\end{array}\right) \frac{1}{q+k+1}, \\
& =\sum_{k=0}^{n}\left(\begin{array}{c}
q \\
n-k
\end{array}\right)\left(\begin{array}{c}
q+k-1 \\
k
\end{array}\right) \frac{(-1)^{n-k}}{q+k+1}
\end{aligned}
$$

Lemma 2 The coefficient $c_{n}$ in the expression for $u_{1}(w)$ given in (33), can be written as

$$
\begin{aligned}
& c_{n}=-\frac{q}{q+1} \frac{1}{\left(\begin{array}{c}
n+q+1 \\
n
\end{array}\right)} \equiv-\frac{q \Gamma(n+1) \Gamma(q+1)}{\Gamma(q+n+2)}, \quad n \geq 1 \\
& c_{0}=\frac{1}{q+1} .
\end{aligned}
$$

Proof. See appendix.

We now evaluate the integrals (31) and (32). We first consider (31), and writing $u_{1}(w)=q w \sum_{k=1}^{\infty} c_{k} w^{k}$ we find:

$$
\begin{aligned}
\int_{h}^{1-h} \frac{u_{1}(w)}{w^{2}} \mathrm{~d} w & =q \int_{h}^{1-h} c_{0} \frac{1}{w}+\sum_{k=1}^{\infty} c_{k} w^{k-1} \mathrm{~d} w \\
& =q c_{0}[\log w]_{h}^{1-h}+q \sum_{k=1}^{\infty}\left[\frac{c_{k}}{k} w^{k}\right]_{h}^{1-h} \\
& =q c_{0}(-\log h)+q \sum_{k=1}^{\infty} \frac{c_{k}}{k}+O(h) .
\end{aligned}
$$

In the appendix we show that

$$
\sum_{k=1}^{\infty} \frac{c_{k}}{k}=-\frac{q}{(q+1)^{2}}
$$

and so, using this result together with (36), we have

$$
\int_{h}^{1-h} \frac{u_{1}(w)}{w^{2}} \mathrm{~d} w=-\frac{q}{q+1} \log h-\frac{q^{2}}{(q+1)^{2}}+O(h) .
$$

Now we consider (32). We expand both $u_{1}(w)$ and $(1-w)^{-1}$ to find:

$$
\int_{h}^{1-h} \frac{u_{1}(w)}{w(1-w)} \mathrm{d} w=q \int_{h}^{1-h}\left(\sum_{k=0}^{\infty} w^{k}\right)\left(\sum_{k=0}^{\infty} c_{k} w^{k}\right) \mathrm{d} w
$$


Resonance bifurcations from robust homoclinic cycles

$$
\begin{aligned}
& =q \int_{h}^{1-h} \sum_{k=0}^{\infty} d_{k} w^{k} \mathrm{~d} w, \\
& =q\left[\sum_{k=0}^{\infty} \frac{d_{k}}{k+1} w^{k+1}\right]_{h}^{1-h}, \\
& =q \sum_{k=0}^{\infty} \frac{d_{k}}{k+1}+O(h),
\end{aligned}
$$

where we have defined $d_{k}=\sum_{j=0}^{k} c_{j}$. We evaluate $d_{k}$ in the appendix to find:

$$
\sum_{k=0}^{\infty} \frac{d_{k}}{k+1}=\frac{1}{q}
$$

Hence

$$
\int_{h}^{1-h} \frac{u_{1}(w)}{w(1-w)} \mathrm{d} w=1+O(h) .
$$

Finally, we use our expansion of $u_{1}$ to find $u_{1}(h)=q h \sum_{k=0}^{\infty} c_{k} h^{k}$, and so

$$
\log \left(1-\frac{\beta u_{1}(h)}{h}\right)=-\beta q c_{0}+O(\beta h)=-\beta \frac{q}{1+q}+O(\beta h)
$$

and

$$
\log \left(1-\frac{\beta u_{1}(h)}{1-h}\right)=O(\beta h) .
$$

Therefore, substituting (37), (38), (39) and (40) into (30) we have

$$
\begin{aligned}
2 A=-\beta \frac{q}{q+1}+q(2 s-\beta) \log h-\beta q(n-1)+\beta(q+1)\left(\frac{q}{q+1}(-\log h)-\frac{q^{2}}{(q+1)^{2}}\right) & \\
& +O(\beta h)+O\left(\beta^{2}\right), \\
= & 2 q(s-\beta) \log h+\beta\left(-\frac{q}{q+1}-n q+q-\frac{q^{2}}{q+1}\right)+O(\beta h)+O\left(\beta^{2}\right),
\end{aligned}
$$

which simplifies dramatically to give

$$
A=q(s-\beta) \log h-\frac{\beta n q}{2}+O(\beta h)+O\left(\beta^{2}\right) .
$$

By definition, as we approach the bifurcation point $s \rightarrow \beta$, and so, evaluating $A$ at the bifurcation point we obtain to leading order

$$
A \rightarrow-\frac{\beta n q}{2}<0,
$$

and hence the bifurcation is found to be supercritical for all values of $n \geq 4$ and $q=1 / c$. Note that although our calculation only computes $A$ to leading order in $\beta$, since the criticality only depends on the sign of $A$, the bifurcation will be supercritical whenever $\beta$ is sufficiently small. 


\section{Bifurcating periodic orbits}

In this section we discuss the evolution of the unique stable periodic orbit produced in the resonance bifurcation for the system considered in section 4 .

First we use the calculation of the return map to derive an approximate expression for the period of the orbit. We emphasise that the analytic result contains no adjustable parameters. Referring back to the calculation of the return map, combining equations (9) for the time spent in a neighbourhood of an equilibrium on the cycle with (18) which gives the leading-order relation between the location of the fixed point of the Poincaré map and the coefficients $C_{j}$, we obtain the following expression for the period $P$ of the periodic orbit, as usual neglecting the time spent moving between neighbourhoods of the equilibria on the cycle:

$$
P \equiv n T=-\frac{n}{e} \log \left(C^{1 /(1-\delta)}\right),
$$

where $\delta=(c+s) / e$ and $C=\prod_{j=l+2}^{n} \tilde{C}_{j}$ as before. Substituting $\log C \equiv A=-n \beta q / 2$ where $\beta=e-c>0$ and $q=1 / c$ this expression simplifies to yield

$$
P=\frac{n}{e} \frac{n \beta q}{2(1-\delta)}=\frac{n^{2}(e-c)}{2 c(e-c-s)} .
$$

This expression has been derived near the resonance bifurcation (i.e. $|e-c-s| \ll 1$ ), in the case that $|e-c| \ll 1$ and therefore is expected to be asymptotically correct in the limits of small $s$ and small $e-c$.

We compare the analytic result (42) with numerical integrations of (17). Our numerical integrations are carried out with a standard 4th order Runge-Kutta scheme, with the return times $P_{1}, P_{2}, \ldots$ to the cross-section $x_{1}=0.2$ computed by linearly interpolating points on the trajectory that lie on either side of the cross-section. Since trajectories may be assumed to converge exponentially to the periodic orbit (discounting the possibility that it is nonhyperbolic) a highly accurate extrapolation of the true period

of the orbit may be obtained by employing the ansatz $P_{k}=P+b_{0} \mathrm{e}^{-b_{1} k}$ and eliminating the coefficients (which are supposed to be constant) $b_{0}$ and $b_{1}$ by considering a set of three return times $P_{k}, P_{k+m}$ and $P_{k+2 m}$. This leads to the extrapolation formula

$$
P=\frac{P_{k+2 m} P_{k}-P_{k+m}^{2}}{P_{k+2 m}-2 P_{k+m}+P_{k}},
$$

which we find to give excellent results, even when the computed return times $P_{j}$ used in (43) are far from the true (ultimate) period $P$.

Figure 1 confirms the accuracy of the approximation (42), showing the period $P$ as a function of $e-c-s$ for four different values of $s$ in the case $n=4$ (where there is only a single transverse eigenvalue). The excellent agreement for $s=0.01$ (the solid curve and diamond symbols) and $s=0.1$ (dashed curve and triangle symbols) shows that when $0<s \ll c$ the approximate expression (42) is extremely accurate. Figure 2 confirms the accuracy in the limit $0<s \ll c$ when $c$ is varied at fixed $s$; the data for $c=1$ are most accurately approximated by (42). Finally, figure 3 illustrates the dependence of $P$ on $n$ as being $P \sim n^{2}$ - not a scaling that one might intuitively have proposed. For this 


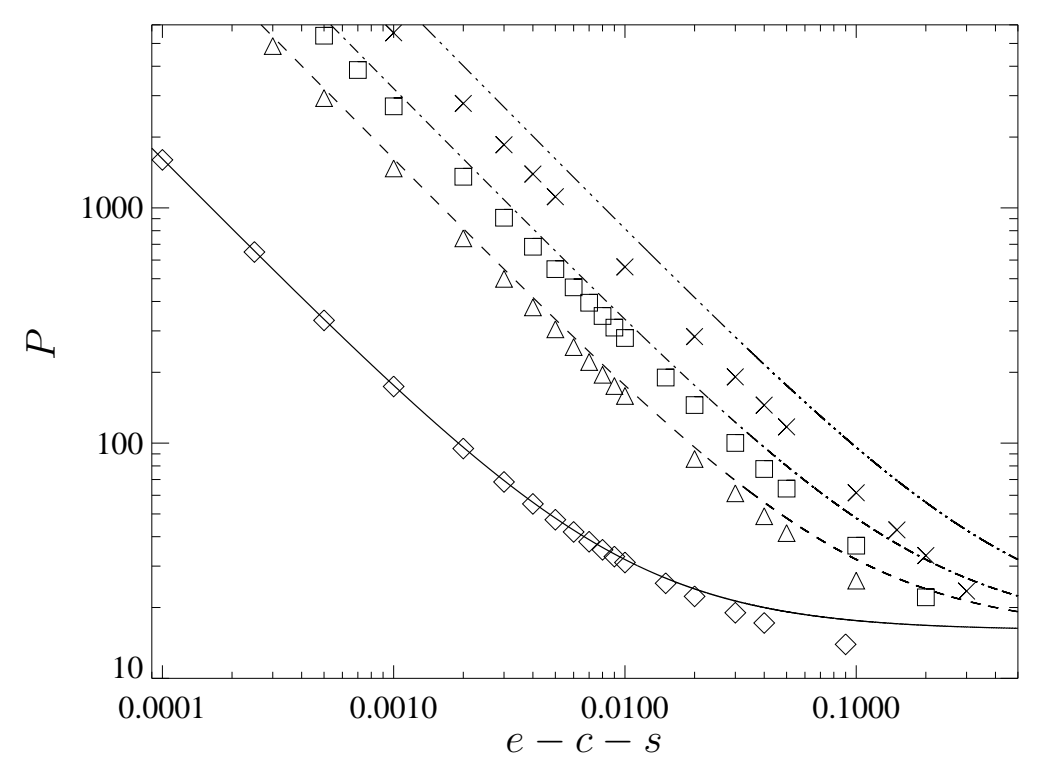

Figure 1. Orbit period $P$ plotted against the eigenvalue combination $e-c-s$ for the periodic orbit created in the resonance bifurcation at $e-c-s=0$. Parameters are $n=4, c=0.5$. Calculations are carried out by varying $e$ for fixed values of $s$. Numerical data and the approximation (42) are shown for four values of $s$, from bottom to top: $s=0.01(\diamond$, solid line); $s=0.1$ ( $\triangle$, dashed line); $s=0.2$ ( $\square$, dash-dotted line); $s=0.5$ ( $\times$, dash-triple-dotted line). The approximation is excellent for small $s$ but systematically overestimates the period for larger $s$.

figure we have for convenience taken $s=0.1$ for $n=4,5,6$ and achieved this by setting $s_{j}=s /(n-3)$ for all $1 \leq j \leq n-3$. We remark that the accuracy of (42) does not appear to vary significantly with either increasing or decreasing $n$.

Further numerical investigations in the case that $s>0$ but allowing one, or more, of the $s_{j}$ to be negative, so that the transverse eigenvalues, $-s_{j}$ are positive, (i.e. when $e-c-s<0$, the homoclinic cycle is only essentially asymptotically stable rather than asymptotically stable) show that a periodic orbit bifurcates at $e-c-s=0$ and has a period that is extremely close to that for the $s_{j}=s /(n-3)$ case as long as $s$ remains much less than $e$. For example, in the case $n=5, s=s_{1}+s_{2}=0.1, c=0.5, e=0.7$ we observed that $P=41.169$ for $s_{1}=s_{2}=0.05$. On increasing $s_{1}$ (and correspondingly decreasing $s_{2}$ so that $s_{1}+s_{2}=0.1$ always) we observed numerically that the period of the periodic orbit remained within $P=41.17 \pm 0.04$ for $0.05 \leq s_{1} \leq 0.65$ before reducing rapidly for larger $s_{1}: P=35.4$ at $s_{1}=0.655$ and $P=30.15$ at $s_{1}=0.66$. It seems reasonable to expect that the shape of the periodic orbit near the equilibria changes substantially when transverse eigenvalues become of the same order as the expanding eigenvalue $e$. We leave a detailed investigation of the dynamics to be the subject of future work. 


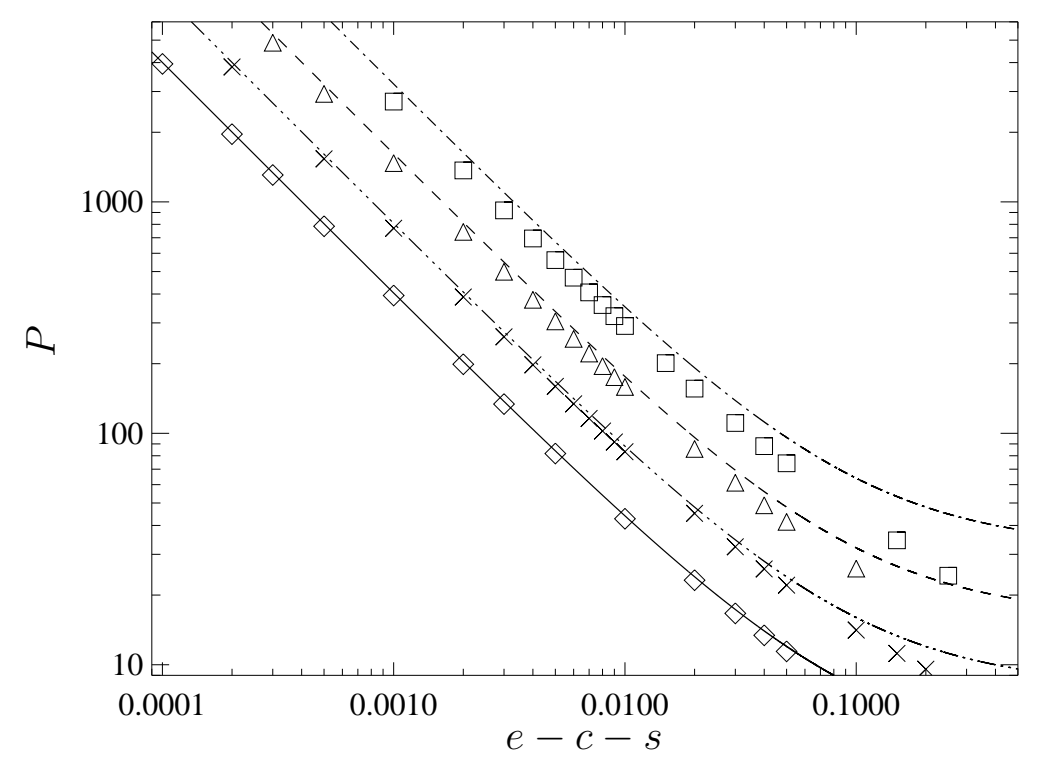

Figure 2. Orbit period $P$ plotted against the eigenvalue combination $e-c-s$ for the periodic orbit created in the resonance bifurcation at $e-c-s=0$. Calculations are carried out by varying $e$ for fixed values of $c$. Parameters are $n=4, s=0.1$. Numerical data and the approximation (42) are shown for four values of $c$, from bottom to top: $c=2(\diamond$, solid line); $c=1$ ( $\times$, dash-triple-dotted line); $c=0.5$ ( $\triangle$, dashed line); $c=0.25$ ( $\square$, dash-dotted line). The approximation is excellent for large $c$ but systematically overestimates the period for smaller $c$. Note that the data for $c=0.5$ $(\triangle$, dashed line) is shown with the same symbols and line style in figures 1 and 3 .

\section{Discussion}

In this paper we have discussed resonance bifurcations in a class of vector fields containing robust homoclinic cycles that connect equilibria on a single group orbit, each lying in an l-dimensional hyperplane in $\mathbb{R}^{n}$. Using the well-known return map technique, and the construction of transition matrices, we proved a general stability result for this class of homoclinic cycles in section 3. We then discussed the resonant bifurcation that occurs when $c+s=e$, where $s$ is the sum of the relevant transverse eigenvalues.

In the case that the equilibria lie on the axes in $\mathbb{R}^{n}$ it is possible to make substantial further progress and compute the period of the bifurcating orbit by integrating along trajectories to explicitly compute the global parts of the return map. To the best of our knowledge, this is the first calculation of its kind.

The calculation rests on the assumption that the sum $s$ of the relevant transverse eigenvalues is small compared to the leading stable and unstable eigenvalues ( $c$ and $e$ respectively). The details of the calculation become algebraically rather complicated, but only involve computing a number of integrals; this is achieved through power series expansions and summations. Unlike most return map calculations where the global 


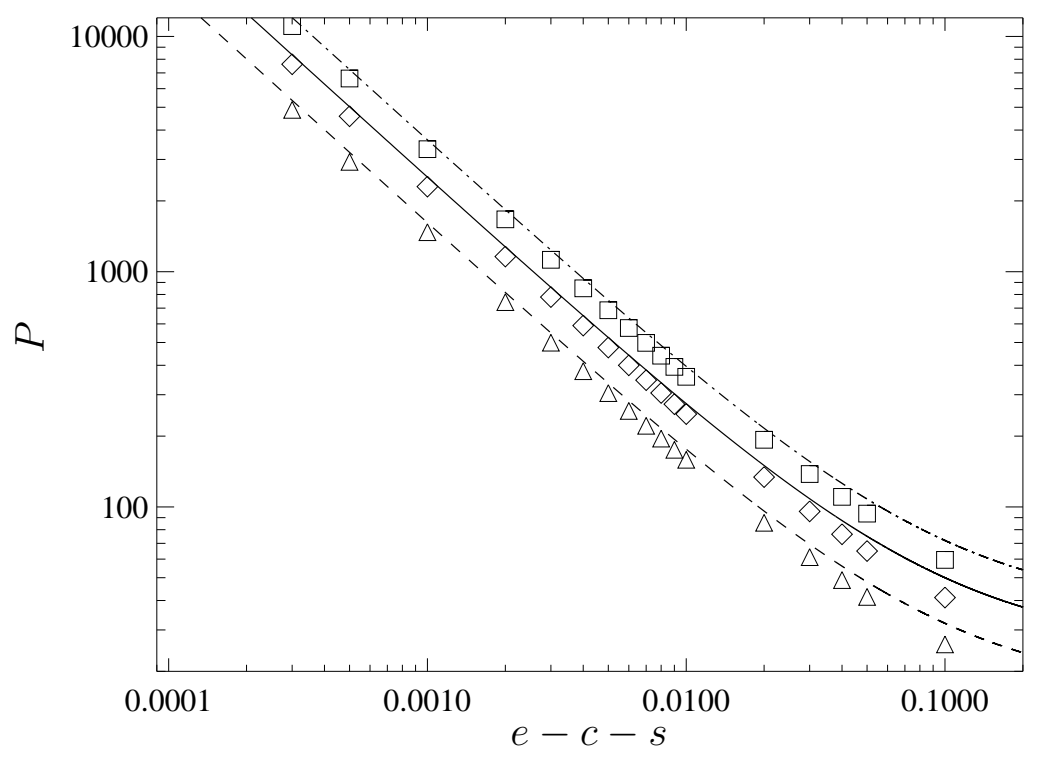

Figure 3. Orbit period $P$ plotted against the eigenvalue combination $e-c-s$ for the periodic orbit created in the resonance bifurcation at $e-c-s=0$. Calculations are carried out by varying $e$ for fixed values of $s=0.1$ and $c=0.5$, keeping all the $s_{j}$ equal as discussed in the text. Numerical data and the approximation (42) are shown for three values of $n$, from bottom to top: $n=4(\triangle$, dashed line); $n=5(\diamond$, solid line); $n=6$ ( $\square$, dash-dotted line). No trend in increasing or decreasing accuracy is observed as $n$ varies. Note that the data for $n=4$ ( $\triangle$, dashed line) is shown with the same symbols and line style in figures 1 and 2 .

parts of the map introduce undetermined coefficients whose magnitudes are rarely known, our results contain no free parameters and are seen to agree well with numerical computations of the period of the bifurcating orbit.

It is of interest that the calculation shows that for this class of systems the resonance bifurcation is always supercritical. It would be of interest to investigate extending the present work to find a correspondingly simple class of examples where the criticality of the bifurcation depended non-trivially on parameters. For instance, in [20] we give an example of a cycle which has a resonant bifurcation, the criticality of which depends on parameters. It would also be interesting to see to what extent the analysis here could be applied to heteroclinic cycles in Lotka-Volterra systems, such as those studied, mainly numerically, by other authors $[14,15,16,17]$.

\section{Appendix}

In this appendix we provide the proof of lemma 2, and the computation of the sums $\sum_{k=1}^{\infty} \frac{c_{k}}{k}$ and $\sum_{k=0}^{\infty} \frac{d_{k}}{k+1}$ which are used in section 4.4 in the computation of $A$. 
Proof of lemma 2 It is trivial to check that $c_{0}=1 /(q+1)$. For $n \geq 1$, let

$$
f(n)=\sum_{k=0}^{n} F(n, k)
$$

where

$$
F(n, k)=\frac{1+q}{q} \frac{(-1)^{n-k+1}}{q+k+1}\left(\begin{array}{c}
q+k-1 \\
k
\end{array}\right)\left(\begin{array}{c}
q \\
n-k
\end{array}\right)\left(\begin{array}{c}
n+q+1 \\
n
\end{array}\right),
$$

that is, $f(n)$ is the right hand side of (34) divided by the right hand side of (35). We will show that $f(n)$ is independent of $n$, and specifically, that $f(n)=1$, which proves the lemma.

Note that $F(n, k)=0$ for $k \geq n+1$. Further, let

$$
R(n, k)=\frac{-k(1+k+q)(k-n+q)(k-1-n-n q)}{q n(1+n)^{2}(1-k+n)}
$$

for $k \neq n+1$, and define $G(n, k)=R(n, k) F(n, k)$. Note that $G(n, n+1)$ is well defined, and $G(n, k)=0$ for $k \geq n+2$. It can be verified that

$$
F(n+1, k)-F(n, k)=G(n, k+1)-G(n, k)
$$

Summing over all $k$ gives

$$
\begin{aligned}
\sum_{k=0}^{\infty} F(n+1, k)-\sum_{k=0}^{\infty} F(n, k) & =\sum_{k=0}^{\infty} G(n, k+1)-\sum_{k=0}^{\infty} G(n, k) \\
\sum_{k=0}^{n+1} F(n+1, k)-\sum_{k=0}^{n} F(n, k) & =\sum_{k=0}^{n} G(n, k+1)-\sum_{k=1}^{n+1} G(n, k)-G(n, 0) \\
f(n+1)-f(n) & =-G(n, 0)=0
\end{aligned}
$$

so $f(n)$ is independent of $n$. It is simple to check that $f(1)=1$, completing the proof.

The following lemma is used a number of times in what follows:

\section{Lemma 3}

$$
\sum_{j=0}^{k} \frac{\Gamma(x+1) \Gamma(j+1)}{\Gamma(x+j+1)}=\frac{x}{x-1}\left(1-\frac{\Gamma(x) \Gamma(k+2)}{\Gamma(x+k+1)}\right)
$$

Proof. We use induction on $k$. It is simple to check that (A.4) holds for $k=1$. Then assuming (A.4) is true for some $k>1$, we find for $k+1$,

$$
\begin{aligned}
\sum_{j=0}^{k+1} \frac{\Gamma(x+1) \Gamma(j+1)}{\Gamma(x+j+1)} & =\sum_{j=0}^{k} \frac{\Gamma(x+1) \Gamma(j+1)}{\Gamma(x+j+1)}+\frac{\Gamma(x+1) \Gamma(k+2)}{\Gamma(x+k+2)} \\
& =\frac{x}{x-1}\left(1-\frac{\Gamma(x) \Gamma(k+2)}{\Gamma(x+k+1)}\right)+\frac{x \Gamma(x) \Gamma(k+2)}{(x+k+1) \Gamma(x+k+1)} \\
& =\frac{x}{x-1}\left(1-\frac{\Gamma(x) \Gamma(k+2)}{\Gamma(x+k+1)}\left(\frac{k+2}{x+k+1}\right)\right) \\
& =\frac{x}{x-1}\left(1-\frac{\Gamma(x) \Gamma(k+3)}{\Gamma(x+k+2)}\right)
\end{aligned}
$$


Corollary 1 For $x>1$,

$$
\sum_{j=0}^{\infty} \frac{\Gamma(x+1) \Gamma(j+1)}{\Gamma(x+j+1)}=\frac{x}{x-1}
$$

Proof. The required result is equivalent to showing that

$$
\lim _{k \rightarrow \infty} \frac{\Gamma(x) \Gamma(k+3)}{\Gamma(x+k+2)}=0 .
$$

To show this we write the combination of Gamma functions as a Beta function:

$$
\frac{\Gamma(x) \Gamma(k+3)}{\Gamma(x+k+2)}=(k+2) B(k+2, x)=(x-1) B(k+3, x-1),
$$

where we define the usual Beta function

$$
B(p, q)=\int_{0}^{1} t^{p-1}(1-t)^{q-1} d t=\int_{0}^{\infty} \frac{s^{q-1}}{(1+s)^{p+q}} d s
$$

making the change of variable $1+s=t^{-1}$, see [21]. The last equality in (A.5) can be derived in a straightforward manner by integrating by parts the first integral expression in (A.6) for $B(p, q)$. Then we have

$$
\begin{aligned}
(x-1) B(k+3, x-1) & =\int_{0}^{\infty} \frac{(x-1) s^{x-2}}{(1+s)^{x+k+2}} d s<\int_{0}^{\infty}(x-1)(1+s)^{-k-4} d s \\
& <-\left.\frac{(x-1)(1+s)^{-k-3}}{k+3}\right|_{s=0} ^{\infty}=\frac{x-1}{k+3},
\end{aligned}
$$

which clearly tends to zero as $k \rightarrow \infty$ for any fixed $x>1$.

We are now able to compute the following two sums which are used in section 4.4 . Firstly, consider

$$
\begin{aligned}
\sum_{k=1}^{\infty} \frac{c_{k}}{k}=-q & \sum_{k=1}^{\infty} \frac{\Gamma(k+1) \Gamma(q+1)}{k \Gamma(q+k+2)} \\
& =-\frac{q}{(q+2)(q+1)} \sum_{k=1}^{\infty} \frac{\Gamma(k) \Gamma(q+3)}{\Gamma(q+k+2)}=-\frac{q}{(q+2)(q+1)} \sum_{k=0}^{\infty} \frac{\Gamma(k+1) \Gamma(q+3)}{\Gamma(q+k+3)} \\
& =-\frac{q}{(q+2)(q+1)} \frac{q+2}{q+1}=-\frac{q}{(q+1)^{2}} .
\end{aligned}
$$

Now consider

$$
\begin{aligned}
d_{k} & =\sum_{j=0}^{k} c_{j}, \\
& =c_{0}-q \sum_{j=1}^{k} \frac{\Gamma(j+1) \Gamma(q+1)}{\Gamma(q+j+2)} \\
& =\frac{1}{1+q}+\frac{q \Gamma(q+1)}{\Gamma(q+2)}-q \sum_{j=0}^{k} \frac{\Gamma(j+1) \Gamma(q+1)}{\Gamma(q+j+2)}, \\
& =\frac{1}{1+q}+\frac{q}{1+q}-\frac{q}{q+1} \sum_{j=0}^{k} \frac{\Gamma(j+1) \Gamma(q+2)}{\Gamma(q+j+2)},
\end{aligned}
$$




$$
\begin{aligned}
& =1-\frac{q}{(q+1)} \frac{(q+1)}{q}\left(1-\frac{\Gamma(q+1) \Gamma(k+2)}{\Gamma(q+k+2)}\right), \\
& =\frac{\Gamma(q+1) \Gamma(k+2)}{\Gamma(q+k+2)},
\end{aligned}
$$

so that the second sum can be computed to be

$$
\begin{aligned}
\sum_{k=0}^{\infty} \frac{d_{k}}{k+1} & =\sum_{k=0}^{\infty} \frac{\Gamma(q+1) \Gamma(k+1)}{\Gamma(q+k+2)} \\
& =\frac{1}{q+1} \sum_{k=0}^{\infty} \frac{\Gamma(q+2) \Gamma(k+1)}{\Gamma(q+k+2)} \\
& =\frac{1}{q+1} \frac{q+1}{q}=\frac{1}{q}
\end{aligned}
$$

\section{Acknowledgements}

CMP acknowledges support from the University of Auckland Research Council. JHPD currently holds a University Research Fellowship from the Royal Society. Preliminary work this topic was partially funded by the Nuffield Foundation through an Undergraduate Research Bursary for Miss Yin Lin (grant number 2006/33001) and by Newnham College, Cambridge.

\section{References}

[1] Krupa M 1997 Robust heteroclinic cycles J. Nonlinear Sci. 7 129-176

[2] Field M 1996 Lectures on bifurcations, dynamics and symmetry Pitman Research Notes in Mathematics vol 356 London: Longman Scientific and Technical

[3] Krupa M and Melbourne I 1995 Asymptotic stability of heteroclinic cycles in systems with symmetry Ergod. Th. ES Dynam. Sys. 15 121-147

[4] Krupa M and Melbourne I 2004 Asymptotic stability of heteroclinic cycles in systems with symmetry, II Proc. Roy. Soc. Edinburgh A 134A 1177-1197

[5] May R and Leonard W 1975 Nonlinear aspects of competition between three species SIAM J. Appl. Math. 29 243-253

[6] Hofbauer J 1994 Heteroclinic Cycles in Ecological Differential Equations Tatra Mountains Math. Publ. 4 105-116

[7] Hofbauer J and Sigmund K 1998 Evolutionary Games and Population Dynamics CUP

[8] Aguiar M, Ashwin P, Dias A, and Field M 2009 Robust heteroclinic cycles in coupled cell systems: Identical cells with asymmetric inputs. Preprint.

[9] Jones C A and Proctor M R E 1987 Stong spatial resonance and travelling waves in Bénard convection Physics Letters A 121 224-228

[10] Armbruster D, Guckenheimer J and Holmes P 1988 Heteroclinic cycles and modulated traveling waves in systems with $O(2)$ symmetry Physica D 29 257-282.

[11] Guckenheimer J and Holmes P 1988 Structurally stable heteroclinic cycles Math. Proc. Camb. Phil. Soc. 103 189-192

[12] Melbourne I 1991 An example of a non-asymptotically stable attractor Nonlinearity 4 835-844

[13] Driesse R and Homburg A J 2009 Essentially asymptotically stable homoclinic networks To appear in Dynamical Systems 
[14] Rabinovich M, Volkovskii A, Lecanda P, Huerta R, Abarbanel H D I and Laurent G 2001 Dynamical encoding by networks of competing neuron groups: winnerless competition. Phys. Rev. Lett. 87 068102

[15] Venaille A, Varona P and Rabinovich M 2005 Synchronization and coordination of sequences in two neural ensembles. Phys. Rev. E 71061909

[16] Rabinovich M I, Varona P, Selverston A I and Abarbanel H D I 2006 Dynamical principles in neuroscience. Rev. Mod. Phys. 78 1213-1265

[17] Rabinovich M I, Huerta R, Varona P and Afraimovich V S 2008 Transient cognitive dynamics, metastability and decision making. PLoS Comp. Biol. 4 e1000072

[18] Chossat P, Krupa M, Melbourne I and Scheel A 1997 Transverse bifurcations of homoclinic cycles Physica D 100 85-100

[19] Field M and Swift J 1991 Stationary bifurcation to limit cycles and heteroclinic cycles Nonlinearity 4 1001-1043

[20] Postlethwaite C M and Dawes J H P 2006 A codimension two resonant bifurcation from a heteroclinic cycle with complex eigenvalues Dynamical Systems An International Journal 21(3) $313-336$

[21] Whittaker E T and Watson G N 1927 A Course of Modern Analysis. CUP, 4th edition. 